\title{
Quantization of Gravitationally Bound Systems
}

\author{
Michael Fil'chenkov* and Yuri Laptev
}

Institute of Gravitation and Cosmology, RUDN University, 6 Miklukho-Maklay Str., 117198 Moscow, Russia; yplaptev@rambler.ru

* Correspondence: fmichael@mail.ru

Citation: Fil'chenkov, M.; Laptev, Y. Quantization of Gravitationally Bound Systems. Universe 2021, 7, 30. https://doi.org/10.3390/ universe7020030

Academic Editor: Vladimir M. Mostepanenko and Alexei A. Starobinsky

Received: 28 November 2020

Accepted: 27 January 2021

Published: 30 January 2021

Publisher's Note: MDPI stays neutral with regard to jurisdictional claims in published maps and institutional affiliations.

Copyright: (c) 2021 by the authors. Licensee MDPI, Basel, Switzerland. This article is an open access article distributed under the terms and conditions of the Creative Commons Attribution (CC BY) license (https:// creativecommons.org/licenses/by/ $4.0 /)$.

\begin{abstract}
Some of the approaches to quantization in gravity theory concerning gravitationally bound systems are considered. Grades of quantization applicable to these systems have been classified in terms of quantum mechanics, quantum field theory, and quantum geometrodynamics. Energy levels for the graviatom, Lemaitre's atom, quantum gravitational collapse have been calculated, and relationships for the masses of bound system components, as well as Universe's birth probabilities, are presented to exemplify the properties of gravitationally bound systems. Objects and processes in them have been analyzed to construct quantum models of compact astrophysical objects and the early Universe.
\end{abstract}

Keywords: quantum gravity; quantization in a given space-time; space-time quantization; graviatoms; Lemaître atom; gravitational collapse

\section{Introduction}

There exist two approaches [1-3] towards quantizing gravity itself: a perturbative field-theoretical approach, wherein gravitons are introduced, and a non-perturbative geometrical approach.

The perturbative quantum gravity proves to be non-renormalizable in the general case. Weak gravitational fields are usually considered in the framework of tensor field theory in Minkowski space. Non-perturbative loop quantum gravity generalizes quantum geometrodynamics at scales that are comparable with the Planckian length. The geometrical quantities are operators having discrete eigenvalues; hence, there are no divergences in loop quantum gravity.

Some approaches to quantization in gravity theory are well developed. Especially, it concerns quantum field theory in curved space-time [4]. Others have not found a wide application. They concern quantum mechanics in a gravitational field [5], quantum gravitational collapse [6], and quantum cosmology [7], when we deal with gravitationally bound systems, which are a subject of our investigation. Below, different features of graviatoms and Lemaître's atom as well as the Universe's birth have been clarified.

The article is organized, as follows. Section 2 presents basic concepts and definitions of quantization for the objects and processes in gravitationally bound systems that are considered in the subsequent sections. In Section 3, the quantum equations representable in Schrödinger's type form are presented. Section 4 exemplifies the energies of gravitationally bound systems. In Section 5 the relation between the masses of primordial black holes and particles captured by them are considered. Section 6 is devoted to the probabilities of Universe's birth, as a result of quantum fluctuation or gravitational collapse. Finally, section 7 presents the general properties of gravitational bound quantum systems and their significance in solving the gravity quantization problem.

This paper reports our results (Sections 3-6) concerning quantization in gravity theory, which are significant for bound quantum systems. They promise a major advance in understanding the general pattern of a consistent theory of gravity quantization. 


\section{Quantization in Gravity Theory}

Gravity is considered in the framework of general relativity and Newtonian theory, and quantum theory - in terms of quantum mechanics and quantum field theory [8].

The grades of quantization in a given space-time or Newtonian gravitational field are as follows: quantum mechanics in the Newtonian gravitational field with relativistic corrections and quantum theory of extraneous fields in curved space-time.

Nonrelativistic quantum mechanics in curved space-time results in Schrödinger's equation in flat space with the Newtonian gravitational potential $\phi_{N}$ [5]:

$$
i \hbar \frac{\partial \psi}{\partial t}=-\frac{\hbar^{2}}{2 m} \Delta \psi+m \phi_{N} \psi,
$$

where $\psi$ is the wave function and $m$ the particle mass.

The gravitational-field and space-time quantization includes: weak gravitational field quantization involving gravitons, quantum geometrodynamics for geometry as a whole, and loop quantum gravity for space-time itself.

Quantum geometrodynamics is considered in space of three-geometries using WheelerDeWitt's equation [9]:

$$
l_{p l}^{4} G_{i j k l} \frac{\delta^{2} \psi}{\delta \gamma_{i j} \delta \gamma_{k l}}+{ }^{3} R \psi=0,
$$

where $\gamma_{i j}$ is the spatial metric, $G_{i j k l}$ the supermetric, and ${ }^{3} R$ the three-curvature,

$$
l_{p l}=\sqrt{\frac{\hbar c}{G}}
$$

is the Planckian length.

In the quasiclassical limit $\psi \sim e^{\frac{i S}{\hbar}}$, where the action $S$ satisfies Peres' equation [10]

$$
\frac{G^{2}}{c^{6}} G_{i j k l} \frac{\delta S}{\delta \gamma_{i j} \delta \gamma_{k l}}-{ }^{3} R \psi=0
$$

The objects and processes in gravitationally bound systems correspond to these grades of quantization, as follows: graviatoms (this term was first introduced in our works [11,12]), which comprise miniholes (i.e., primordial black holes) that are capable of capturing elementary particles [13,14], the Universe's birth from de Sitter's vacuum, quantum gravitational collapse, and particle creation near horizons and in the early Universe .

Below, the energy levels for the graviatom, Lemaitre's atom [15] (simulating quantum early Universe [7]) and quantum gravitational collapse, as well as masses of bound system components and Universe's birth probabilities are presented using solutions to Schrödinger's type equations.

\section{Schrödinger's Type Equations}

The quantum equations for a hydrogen-like graviatom and Lemaitre's atom, describing the early Universe with stiff matter, as well as quantum gravitational collapse, are representable in Schrödinger's type form.

In the case of graviatom to the Newtonian potential energy $m \phi_{N}$, a term

$$
U_{s}=\frac{G M q^{2}}{2 c^{3} r^{2}}
$$

that allows for DeWitt's self-action for a charge $q$ [16], should be added. 
The radial wave functions for the graviatom $R_{p l}=\frac{\chi_{p l}}{r}$, where $p=n-s-1, n$ and $l$ represent the principal and orbital quantum numbers respectively, with

$$
\begin{aligned}
s(s+1) & =\frac{2 m A}{\hbar^{2}}+l(l+1), \\
A & =\frac{m c^{2} r_{q} r_{g}}{4},
\end{aligned}
$$

where

$$
r_{g}=\frac{2 G M}{c^{2}}
$$

is the gravitational radius of the minihole, $M$ its mass,

$$
r_{q}=\frac{q^{2}}{m c^{2}}
$$

is the classical radius of a particle with the mass $m$ and charge $q$.

Equation (6) is reducible in two limiting cases to formulae, as follows: $s \approx l$ for $\frac{2 m A}{\hbar^{2}} \ll l(l+1)$ is valid for hydrogen-like graviatoms and $s \approx \sqrt{\frac{2 m A}{\hbar}}$ is valid for nuclei with masses $m=2 Z m_{p}$ and charges $Z>10^{4}$, which cannot not exist, since they convert into an electroneutral plasma consisting of electrons, protons, and neutrons [17]. Thus, hydrogen-like graviatoms are considered, only to whose relativistic corrections the quantity A may contribute.

The function $\chi_{p l}$ satisfies the equation [18]

$$
\frac{d^{2} \chi_{p l}}{d r^{2}}-\frac{2 m}{\hbar^{2}}\left[U_{e f f}(r)-E_{g a}\right] \chi_{p l}=0,
$$

where

$$
U_{e f f}(r)=\frac{m c^{2} r_{q} r_{g}}{4 r^{2}}-\frac{m c^{2} r_{g}}{2 r}+\frac{\hbar^{2} l(l+1)}{2 m r^{2}},
$$

which has the form of Kratzer's potential for a diatomic molecule [19].

The wave function $\psi(a)$ of Lemaître's atom satisfies the equation [20]

$$
\frac{d^{2} \psi}{d a^{2}}-\frac{2 m_{p l}}{\hbar^{2}}\left[U(a)-E_{L a}\right] \psi=0,
$$

where the potential energy, which was obtained in the framework of McCrea-Milne's classical cosmological model [21], replacing the generalized momentum $p=\frac{d a}{d \eta}$ by the operator $\hat{p}=\frac{l_{p l}^{2}}{i} \frac{d}{d a}$, has the form

$$
U(a)=\frac{m_{p l} c^{2}}{2 l_{p l}^{2}}\left(k a^{2}-\frac{8 \pi G \varepsilon a^{4}}{3 c^{4}}\right),
$$

$a$ is the scale factor, $a d \eta=c d t, \eta$ conformal, and $t$ synchronous time, respectively, $k=0, \pm 1$ the model parameter,

$$
m_{p l}=\sqrt{\frac{\hbar c}{G}}
$$

is the Planckian mass.

The synchronous time in McCrea-Milne's classical model

$$
t=\frac{m_{p l}}{l_{p l}} \int \frac{a d a}{\sqrt{2 m_{p l}\left[E_{L a}-U(a)\right]}}
$$


proves to have been programmed in a quantum model.

Consider a multicomponent medium, for which the energy density

$$
\varepsilon=\varepsilon_{0} \sum_{u} B_{u}\left(\frac{r_{0}}{a}\right)^{u},
$$

where

$$
\frac{1}{r_{0}^{2}}=\frac{8 \pi G \varepsilon_{0}}{3 c^{4}},
$$

$r_{0}$ is de Sitter's horizon, $\varepsilon_{0}$ the energy density of de Sitter's vacuum, and $u=3(1+w)$ with $w$ entering in the formula for the pressure $p=w \varepsilon$ satisfying the barotropic equation of state, providing the normalization condition

$$
\sum_{u} B_{u}=1
$$

is valid, where $B_{u}$ is the contribution of the u-th component on de Sitter horizon.

The cosmological medium has the components, as follows:

1. phantom matter for $u<0$,

2. de Sitter's vacuum for $u=0$,

3. domain walls for $u=1$,

4. cosmic strings for $u=2$,

5. dust for $u=3$,

6. radiation for $u=4$,

7. perfect gas for $u=5$,

8. ultrastiff matter for $u=6$, and

9. ekpyrotic matter for $u>6$.

Lemaitre's atom simulating the early Universe is considered in the framework of quantum cosmology, while using Wheeler-DeWitt's equation of quantum geometrodynamics (2) in the superspace of three-geometries that are reducible to Schrödinger's type Equation (12) in minisuperspace of scale factors, which is being solved while using the formalisms of quantum mechanics. It is in our works $[20,22]$ that the quantum cosmology for mullticomponent model has been considered.

In the case of stiff matter

$$
U(a)=\frac{m_{p l} c^{2}}{2 l_{p l}^{2}}\left(\frac{B_{5} r_{0}^{3}}{a}+\frac{B_{6} r_{0}^{4}}{a^{2}}\right),
$$

where $B_{5}$ and $B_{6}$ are the contributions of perfect gas and ultrastiff matter, respectively, to the total energy density on de Sitter's horizon,

$$
E_{L a}=\frac{m_{p l} c^{2}}{2}\left(\frac{r_{0}}{l_{p l}}\right)^{2} B_{4}
$$

where $B_{4}$ is the contribution of radiation to the total energy density on de Sitter's horizon.

The wave function describing quantum gravitational collapse $\psi(\gamma)$, providing $B_{0}=1$ and $\left|\gamma-\gamma_{0}\right| \ll 1$, satisfies the equation [6]

$$
\frac{d^{2} \psi}{d \gamma^{2}}+\left(\frac{r_{0}}{l_{p l}}\right)^{4}\left[\gamma_{0}^{4}+\frac{B_{5}}{\gamma-\gamma_{0}}+\frac{B_{6}}{\left(\gamma-\gamma_{0}\right)^{2}}+B_{4}\right] \psi=0
$$


providing $B_{0}=1$ and $\left|\gamma-\gamma_{0}\right| \ll 1$, where $\gamma=\frac{a}{r_{0}}$. At $\gamma=\gamma_{0}$, the equation of state changes and there arises de Sitter's vacuum [23]. For $\gamma \gg 1$, we have

$$
\frac{d^{2} \psi}{d \gamma^{2}}+\left(\frac{r_{0}}{l_{p l}}\right)^{4} \gamma^{4} \psi=0
$$

Equations (20) and (21) can be joined at $\gamma=\gamma_{0}+1$, if $\gamma_{0} \gg 1, B_{6}=0, B_{4}=-B_{5}$. Figure 1 presents the potential anergy for the quantum gravitational collapse.

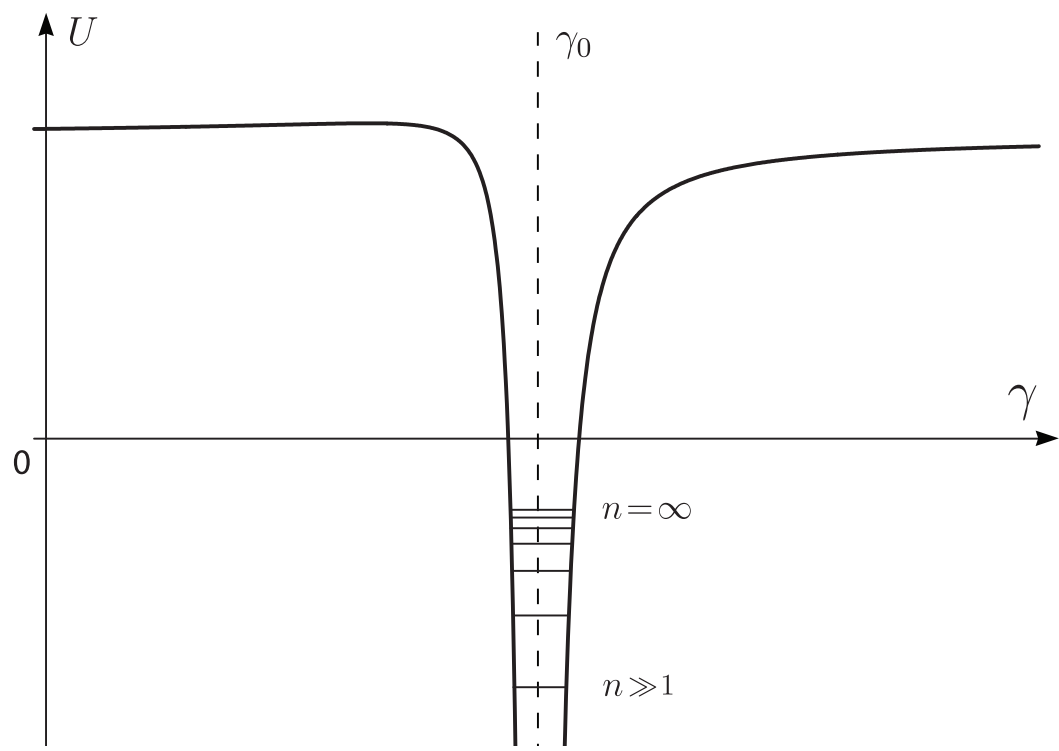

Figure 1. The potential energy for quantum gravitational collapse.

In this case the energy spectrum is given by Formula (21), where $B_{4}$ satisfies the equation

$$
B_{4}^{2}+4 n^{2}\left(\frac{l_{p l}}{r_{0}}\right)^{4}+4 \gamma_{0}^{4} n^{2}\left(\frac{l_{p l}}{r_{0}}\right)^{4}=0 .
$$

Quantum gravitational collapse is analyzed in the framework of quantum cosmology, since the geometry of collapse corresponds with that of Friedmann's contracting model [24].

\section{Energies of Graviationally Bound Quantum Systems}

The graviatom energy levels $[11,12]$ have the form

$$
E_{g a}=-\frac{2 B^{2} m}{\hbar^{2}\left[2 p+1+\sqrt{(2 l+1)^{2}+\frac{8 m A}{\hbar^{2}}}\right]^{2}},
$$

where

$$
B=\frac{m c^{2} r_{g}}{2}
$$

which follows from (10) and (11).

The energy levels of the pre-de-Sitter Universe behaving as a planckeon with the energy of radiation (cf. Lemaitre's atom) in the field of stiff matter [20] are given by the formula

$$
E_{L a}=-\left(\frac{r_{0}}{l_{p l}}\right)^{6} \frac{B_{5}^{2} m_{p l} c^{2}}{8\left[p+\frac{1}{2}+\sqrt{\frac{1}{4}-B_{6}\left(\frac{r_{0}}{l_{p l}}\right)^{4}}\right]^{2}}
$$


where $p=0,1,2 \ldots ; r_{0}$ is de Sitter's horizon, and $B_{5}$ and $B_{6}$ the contribution of perfect gas and ultrastiff matter, respectively, to the total energy density on de Sitter's horizon, which follows from (12)-(14) and (16).

For the graviatoms with

$$
\frac{8 m A}{\hbar} \ll(2 l+1)^{2}
$$

and for the Universe filled with stiff matter with

$$
B_{6}\left(\frac{r_{0}}{l_{p l}}\right)^{6} \ll \frac{1}{4}
$$

we obtain hydrogen-like spectra

$$
E=-\frac{E_{a}}{2 n^{2}},
$$

where $E_{a}$ is the atomic energy unit, which is given by the formula

$$
E_{a}=m c^{2} \alpha_{g}^{2}
$$

for the graviatom, where

$$
\alpha_{g}=\frac{G M m}{\hbar c}
$$

is the gravitational equivalent of the fine structure constant, and the formula

$$
E_{a}=B_{5}^{2}\left(\frac{r_{0}}{l_{p l}}\right)^{6} m_{p l} c^{2}
$$

for Lemaître's atom.

The energy levels in the case of quantum gravitational collapse take values in the interval [6]

$$
-2 \gamma_{0}^{4} \leq B_{4} \leq-\gamma_{0}^{4},
$$

where $B_{4}=-2 \gamma_{0}^{4}$ is the lowest level and $B_{4}=-\gamma_{0}^{4}$ corresponds to the continuum.

\section{System Component Masses}

The masses of miniholes and particles that are captured by them in the graviatom and masses of leptoquarks $m_{X}$ born in the early Universe are related by the same relationship:

$$
M m \sim \frac{1}{2} m_{p l}^{2},
$$

which follows from the existence conditions for hydrogen-like graviatoms [11,12]

$$
\sqrt[3]{\frac{2 b}{9 \pi}}<\alpha_{g}<\frac{1}{\sqrt{2}}
$$

where $b=2.2822$, and the leptoquarks being created at the Compton lengths

$$
\lambda_{c}=\frac{\hbar}{m_{X} c}
$$

of the order of the minihole gravitational radii $r_{g}$.

Inequality (35) means that Bohr's radius of the graviatom

$$
a_{B}^{g}=\frac{\hbar^{2}}{G M m^{2}}
$$


exceeds the minihole gravitational radius $r_{g}$, provided that the Hawking's radiation does not destroy the graviatom, i.e.,

$$
\left|E_{10}\right|>\hbar \omega_{H},
$$

where $\left|E_{10}\right|$ is the energy of particle knocking-out from the first level, $\omega_{H}$ Hawking's radiation frequency

$$
\omega_{H}=\frac{b c^{3}}{4 \pi G M} .
$$

The leptoquarks are created on the exit from under the potential barrier as a result of de Sitter's vacuum decay.

Loop quantum gravity and quantum geometrodynamics are valid at the scales from $l_{p l}$ to $r_{0} \sqrt{k-B_{2}}$. At the scales from $l_{p l}$ to $r_{0} \sqrt{k-B_{2}}$ to $\lambda_{c}$ the quantum field theory in curved space-time is validm changing to general relativity, which holds at the scales exceeding the Compton length $\lambda_{c}$.

\section{Universe's Birth Probabilities}

The probability of a universe birth, as a result of gravitational collapse [6], reads

$$
D \sim \exp \left[-2\left|B_{4}\right|^{3 / 4}\left(\frac{r_{0}}{l_{p l}}\right)^{2}\right],
$$

where $\left|B_{4}\right| \gg 1$.

The probability of the birth of a new universe in another space, as a result of gravitational collapse in our space, equals $D^{2}$, since it is necessary to tunnel through two barriers: the first one in our space and the second in the other (see Figure 2).

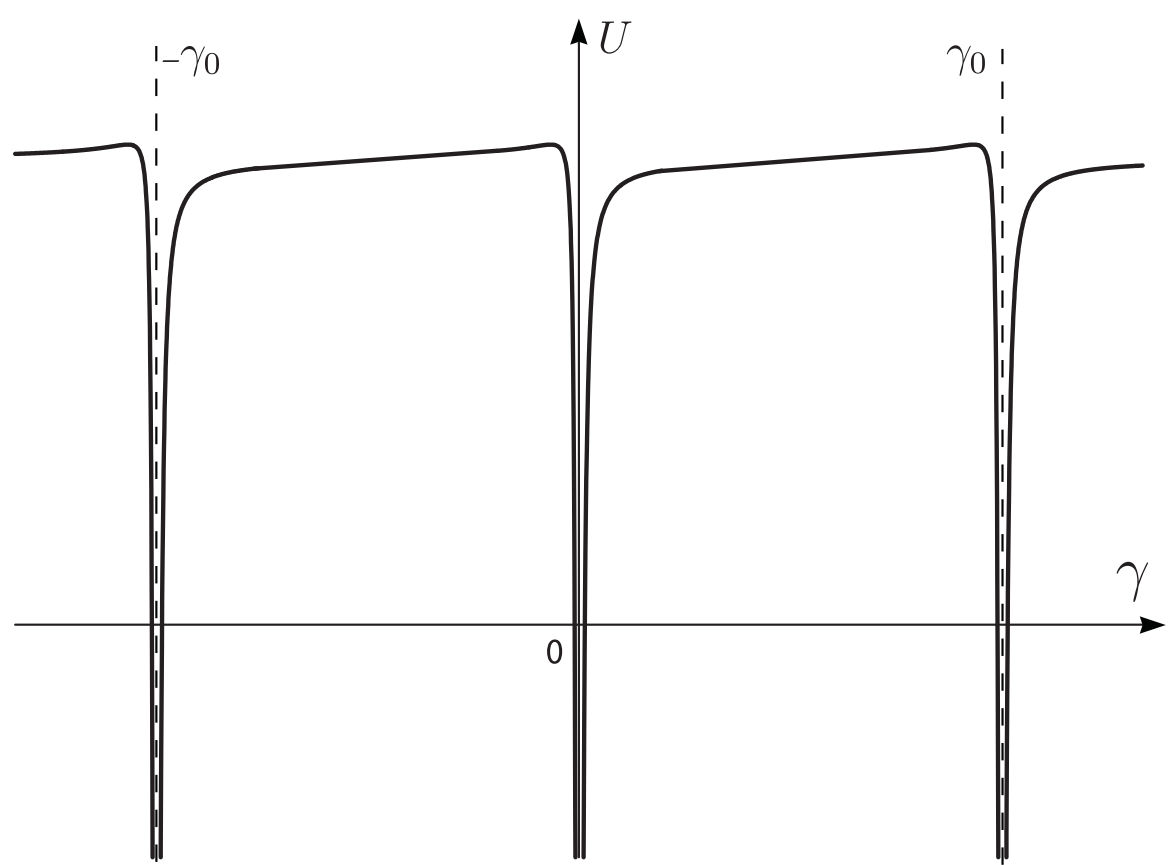

Figure 2. The potential energy for the birth of a new universe, as a result of gravitational collapse.

The probability of a new universe birth, as a result of quantum fluctuation [22], is as follows

$$
D \sim \exp \left[-\frac{2\left(k-B_{2}\right)^{3 / 2}}{3 B_{0}}\left(\frac{r_{0}}{l_{p l}}\right)^{2}\right],
$$


where $B_{0}$ is the contribution of de Sitter's vacuum to the total energy density on de Sitter's horizon, i.e., a tunneling of the planckeon with the energy of radiation through the potential barrier in the case of the potential

$$
U(a)=\frac{m_{p l} c^{2}}{2 l_{p l}^{2}}\left[\left(k-B_{2}\right) a^{2}-\frac{B_{0} a^{4}}{r_{0}^{2}}\right],
$$

where $B_{2}$ is the contribution of cosmic strings to the the total energy density on de Sitter's horizon. For

$$
4 B_{0} B_{4} \sim\left(k-B_{2}\right)^{2}
$$

this case reduces to the previous one if $B_{4} \ll 1$. Thus, the universe's birth as a result of quantum fluctuation is considerably more probable than that for gravitational collapse. In any case, the penetration factor $D$, which describes tunneling through potential barriers, is given by Gamow's formula

$$
D=\exp \left(-\left|\frac{2 r_{0}}{\hbar} \int_{\gamma_{1}}^{\gamma_{2}} \sqrt{m_{p l}(E-U)} d \gamma\right|\right) .
$$

\section{Conclusions}

The general properties of gravitationally bound systems, which are considered in this work, are related to several grades of quantization being simultaneously present at the same quantum objects or processes.

Quantum mechanics and quantum field theory in curved space-time play a part for graviatoms. Hence, there arises a relationship between the masses of miniholes and the particles captured by them.

Schrödinger's type equations describe the graviatom, Lemaître's atom, and quantum gravitational collapse. For the Lemaitre's atom, this follows from Peres' equation containing momentum squared, as well as a possibility of obtaining Friedmann's equation from McCrea-Milne's Newtonian models [21]. It is the similarity of Formulae (24) and (26) for the graviatom and Lemaitre's atom, respectively, that means a fundamental unity between the microworld and the megaworld. The structure of Schrödinger's type equation is not a cause, but an effect, of this similarity.

The relationships for graviatoms and the formation of miniholes and leptoquarks in the early Universe are similar due to the presence of miniholes and the quantum field effects in both cases. A unified formula for the birth of a new universe, as a result of gravitational collapse and quantum fluctuation, is due to both of the processes being based on the tunnel effect.

The gravitationally bound quantum systems cannot be considered within the only approach, which may be a manifestationm of the general pattern of a future theory [25], wherein one succeeds in a transition to the next stage of solving the gravity quantization problem.

The consideration of gravitationally bound quantum systems may play a part in the creation of a consistent theory of gravity quantization allowing for loop and string cosmology to be combined.

Author Contributions: Conceptualization and methodology, M.F.; investigation, M.F., Y.L.; writingoriginal draft preparation, M.F., Y.L.; writing—review and editing, M.F., Y.L.; visualization, M.F., Y.L. All authors have read and agreed to the published version of the manuscript.

Funding: The publication was prepared with the support of the RUDN University Program 5-100.

Acknowledgments: The authors are grateful to V.M. Mostepanenko for useful discussion and advice.

Conflicts of Interest: The authors declare no conflict of interest. 


\section{References}

1. Rovelli, C. Quantum Gravity; Cambridge University Press: Cambidge, UK, 2004.

2. Kiefer, C. Quantum Gravity; Oxford University Press: Oxford, UK, 2007.

3. Fil'chenkov, M.L.; Laptev, Y.P. Quantum Gravity: From the Micro-World to the Mega-World; Lenand: Moscow, Russia, 2016. (In Russian)

4. $\quad$ Birrell, N.D. Quantum Fields in Curved Space; Cambridge University Press: Cambidge, UK, 1982.

5. Kuchař, K. Gravitation, geometry and nonrelativistic theory. Phys. Rev. D 1980, 22, 1285. [CrossRef]

6. Fil'chenkov, M.L. Quantum gravitational collapse and birth of a new Universe. Phys. Lett. B 1998, 441, 34. [CrossRef]

7. Vilenkin, A. Approaches to quantum cosmology. Phys. Rev. D 1994, 50, 2581. [CrossRef] [PubMed]

8. Fil'chenkov, M.L.; Laptev, Y.P. On the problem of quantization in gravity theory. Metaphysics 2019, 2, 108.

9. DeWitt, B.S. Quantum theory of gravity. Phys. Rev. 1967, 160, 1113. [CrossRef]

10. Peres, A. On Caughy's problem in general relativity II. Nuovo Cimento 1962, 26, 53. [CrossRef]

11. Fil'chenkov, M.L.; Laptev, Y.P. Graviatom dipole radiation. Gravit. Cosmol. 2006, 12, 65.

12. Fil'chenkov, M.L.; Laptev, Y.P. Electromagnetic and gravitational radiation of graviatoms. Astron. Astrophys. Trans. 2006, $25,33$.

13. Dolan, S.R. Instability of the massive Klein-Gordon field on the Kerr spacetime. Phys. Rev. D 2007, 76, 084001. [CrossRef]

14. Dymnikova, I.; Fil'chenkov, M.L. Graviatoms with de Sitter interior. Adv. High Energy Phys. 2013, 2013, 746894. [CrossRef]

15. Lemaître, G. L'hypothèse de l'Atome Primitif. Essai de Cosmogonie; Edition du Griffon: Neufchâtel, France, 1946.

16. DeWitt, C.M.; DeWitt, B.S. Falling charges. Physics 1964, 1, 3. [CrossRef]

17. Migdal, A.B. Fermion and Biosons in Strong Fields; Nauka: Moscow, Russia, 1978. (In Russian)

18. Bethe, H.A.; Salpeter, E.E. Quantum Mechanics of One- and Two-Electron Atoms; Springer: Berlin, Germany, 1957.

19. Flügge, S. Practical Quantum Mechanics I; Springer: Berlin, Germany, 1971.

20. Fil'chenkov, M.L. The pre-de-Sitter Universe in terms of quantum mechanics. Phys. Lett. B 1995, 354, 208. [CrossRef]

21. McCrea, W.H.; Milne, E.A. Newtonian Universes and the curvature of space. Quart. J. Math. 1934, 5, 73. [CrossRef]

22. Fil'chenkov, M.L. Collapse, birth of the Universe, creation a new universe in the laboratory. Gravit. Cosmol. Suppl. 2000, 6, 37.

23. Gliner, E.B. Algebraic properties of the energy-momentum tensor and vacuum-like states of matter. JETP 1966, 22, 378.

24. Harrison, B.K.; Thorne, K.S.; Wakano, M.; Wheeler, J.A. Gravitation Theory and Gravitational Collapse; The University of Chicago Press: Chicago, IL, USA, 1965.

25. Mielczarek, J.; Szydlowski, M. Universe from vacuum in loop-string cosmology. J. Cosmol. Astropart. Phys. 2008, 808, 14. [CrossRef] 